Journal Club

Editor's Note: These short, critical reviews of recent papers in the Journal, written exclusively by graduate students or postdoctoral fellows, are intended to summarize the important findings of the paper and provide additional insight and commentary. For more information on the format and purpose of the Journal Club, please see http://www.jneurosci.org/misc/ifa_features.shtml.

\title{
The Roles of Sleep-Wake States and Brain Rhythms in Epileptic Seizure Onset
}

\author{
Omar J. Ahmed ${ }^{1 \star}$ and Sujith Vijayan ${ }^{1,2 *}$ \\ ${ }^{1}$ Department of Neurology, Harvard Medical School and Massachusetts General Hospital, Boston, Massachusetts 02114, and ${ }^{2}$ Department of Mathematics \\ and Statistics, Boston University, Boston, Massachusetts 02215 \\ Review of Sedigh-Sarvestani et al.
}

Epilepsy is an illness of various shapesand horrible.

-Aretaeus of Cappadocia, ancient Greek physician (Temkin, 1994)

Epilepsy is a collection of syndromes affecting more than 50 million people worldwide (Annegers, 2001). Seizures are classified as either generalized or focal, with focal seizures being the most common type in adults (Chang and Lowenstein, 2003). Focal seizures have many different etiologies (e.g., perinatal brain injury, tumors, hemorrhage, abnormal neuronal migration during development) and can be localized to various brain regions (e.g., frontal or temporal lobe). Seizures starting in medial temporal structures such as the hippocampus are the most common kind of focal epilepsy (Chang and Lowenstein, 2003). These focal seizures often spread to other brain regions (secondary generalization), resulting in the loss of consciousness, uncontrolled tonic-clonic muscle movements, and prolonged periods of postseizure fatigue. Exacerbating the problem for patients is the fact that seizures are noto-

Received March 18, 2014; revised April 21, 2014; accepted April 23, 2014. This work was supported by NINDS Grant F32-NS083208 (0.J.A.) and by the National Science Foundation Grant DMS-1042134 (S.V.). We thank Dr. Anne C. Fernandez for contributing the brain sketches used in Figure 1.

${ }^{*} 0$.J.A. and S.V. contributed equally to this work.

Correspondence should be addressed to $0 \mathrm{mar}$ J. Ahmed, Department of

Neurology, Massachusetts General Hospital, 55 Fruit Street, Thier 423, Boston, MA 02114. E-mail: omar.j.ahmed@gmail.com.

DOI:10.1523/JNEUROSCI.1168-14.2014

Copyright $\odot 2014$ the authors $\quad 0270-6474 / 14 / 347395-03 \$ 15.00 / 0$ riously difficult to predict (Litt and Lehnertz, 2002). A clear understanding of seizure warning signs - be they behavioral or electrophysiological—would be a crucial step toward successfully predicting, and subsequently derailing, impending seizures.

Sleep and epilepsy have been recognized as bedfellows since antiquity. Aristotle identified sleep as a useful predictor of certain kinds of seizures, while Hippocrates noted that a lack of sleep could increase the likelihood of seizures (Temkin, 1994). Periods of rapid eye movement (REM) sleep and non-REM (NREM) sleep are interwoven during a sleep bout. During NREM sleep, electroencephalography (EEG) recordings are marked by relatively high voltages and pronounced lowfrequency oscillatory activity. During REM sleep, in contrast, EEG activity resembles the activity seen during awake behavior; it is of relatively low voltage and generally irregular in nature. However, oscillatory activity does appear during REM sleep in certain regions of the brain. For example, theta oscillations are prominent in the rodent hippocampus during REM sleep, just as they are during active wake states (Fig. 1). If sleep is a useful predictor of certain kinds of seizures, is there a specific sleep state and/or oscillatory activity pattern that is most likely to lead to a seizure? This is the question that a recent study by Sedigh-Sarvestani et al. (2014) attempted to answer.

A model of medial temporal lobe epilepsy (MTLE) is produced by injecting tetanus toxin into rat hippocampus. This drug alters neurotransmitter release, upsets the local excitation-inhibition ratio, and leads to chronic seizures (Benke and Swann, 2004). As in human MTLE, the rodent seizures originate focally, secondarily generalize to other parts of the brain, and include a 3-5 $\mathrm{Hz}$ spike-and-wave component (Benke and Swann, 2004).

Sedigh-Sarvestani et al. (2014) used this rodent model to systematically characterize the sleep-wake states most likely to precede epileptic seizures. Using a combination of cortical EEG, hippocampal local field potential (LFP), and accelerometer recordings, the authors semi-automatically classified rats' $24 \mathrm{~h}$ sleep-wake patterns into four nonoverlapping states: awake with prominent 4-12 Hz hippocampal theta oscillations (wake- $\theta$ ); awake with wide-band hippocampal oscillatory power (wake-wb); REM sleep with strong hippocampal theta but no evidence of movement on the accelerometer; and NREM sleep with high-amplitude cortical $0.5-4 \mathrm{~Hz}$ delta oscillations. Seizures were automatically quantified using the same LFP and EEG signals. For their analyses, the authors included only secondarily generalized seizures. For each seizure, they asked which of the four sleep-wake states immediately preceded the seizure. The results (summarized in Fig. $1 B$ ) were dramatic: although the rats spent only $6 \%$ of their time in REM sleep, $47 \%$ of their seizures started in this state (Sedigh-Sarvestani et 
al., 2014, their Fig. 3). In contrast, despite rats spending $39 \%$ of their time in NREM sleep, $<7 \%$ of seizures arose directly from NREM sleep. There was also a disparity between the waking states: despite spending $27 \%$ of the day in each of the two awake states, $34 \%$ of seizures started in the wake- $\theta$ state, while only $13 \%$ started from wake-wb.

Clearly, states with pronounced theta oscillations (REM and wake- $\theta$ ) were most likely to lead to seizures (Sedigh-Sarvestani et al., 2014, their Fig. 4). However, not all theta epochs were equally epileptogenic in this rat model of MTLE. The seizure rates during REM and wake- $\theta$ states were very different: 8.3 and 1.4 seizures/h, respectively. In addition, only $19 \%$ of REM episodes led to seizures even though all episodes had clear theta oscillations. To avoid a massive number of false-positive seizure prediction errors, the next step is to determine what makes one sequence of theta oscillations more likely to lead to a seizure than another. The authors presented raw LFP traces as visual evidence of the similarity between REM theta that led to a seizure and REM theta that did not (Sedigh-Sarvestani et al., 2014, their Fig. 6). However, it is possible that there are subtle differences in theta frequency or power between the two conditions. Subtle differences in extracellular rhythmic dynamics might result from slightly different levels of excitatory drive of the local neuronal population. Indeed, as rats increase their running speed, the increased synaptic drive of hippocampal place cells results in higher firing rates and slightly faster LFP theta rhythms (Buzsáki, 2005). It is reasonable to expect that a REM theta epoch that precedes epileptiform activity will be associated with increased drive of hippocampal cells and increased LFP theta power and frequency. Spectral analyses over short time windows might be used in the future to dissect the precise differences between REM theta epochs that lead to seizures and those that do not. Any differences in drive might be confirmed using in vivo whole-cell measurements of the membrane potential during pre-seizure and non-pre-seizure REM theta epochs. Finding such subtle differences in extracellular oscillations reflecting differential intracellular membrane potential dynamics across preseizure and non-pre-seizure states is a crucial prerequisite to useful LFP-based seizure prediction.

Since rodent REM states are, in practice, defined by the presence of pronounced hippocampal theta oscillations,
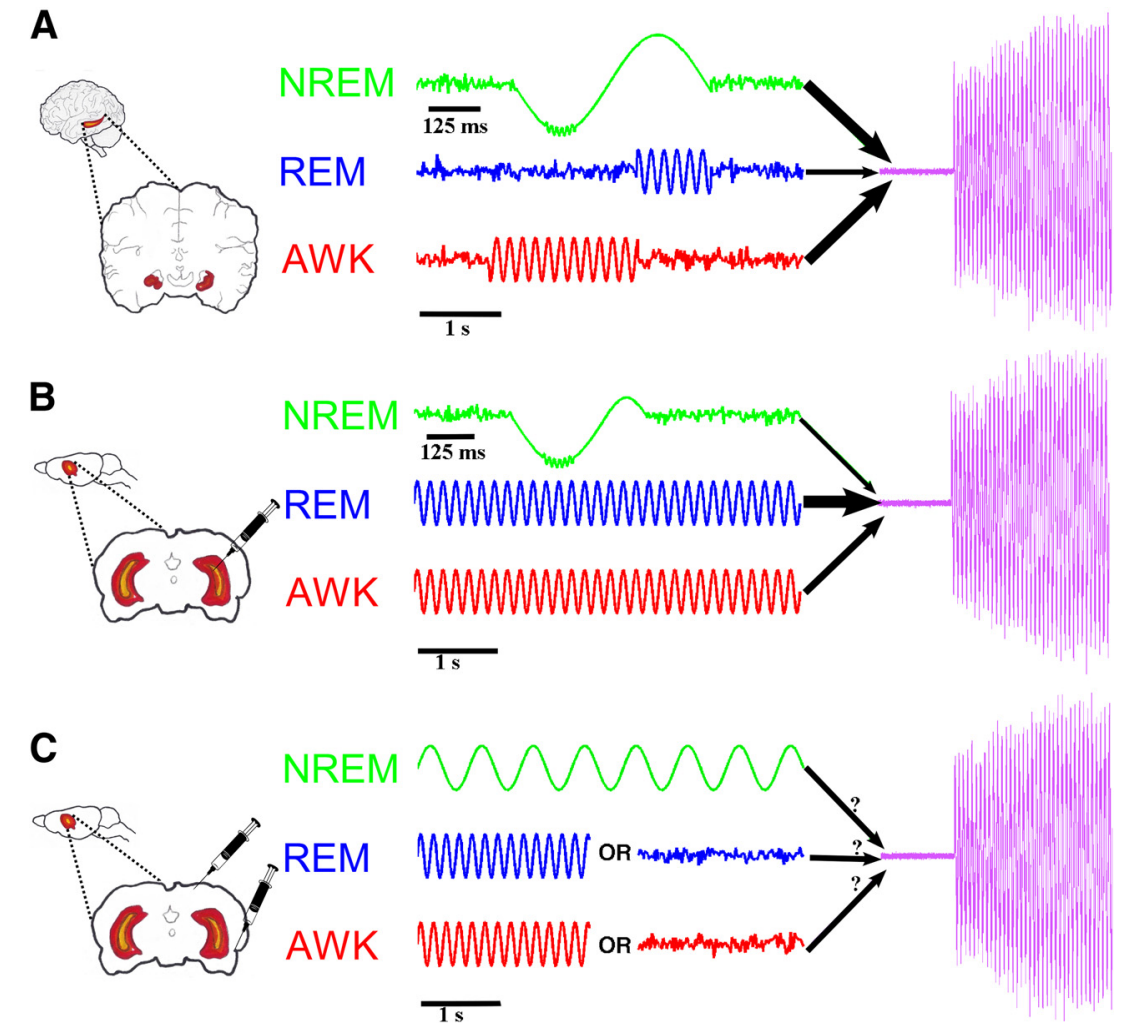

Figure 1. Relationship between sleep-wake states, brain rhythms, and seizure onset across species and regions. $\boldsymbol{A}$, Left, In human MTLE, focal seizures arise from the hippocampus (shown in red/yellow on the left) and related structures. Right, Schematic depiction of hippocampal LFP recordings during NREM sleep (green), REM sleep (blue), and awake (AWK, red) states. NREM sleep is characterized by occasional sharp-wave-ripple complexes, REM states by occasional short-lasting hippocampal theta rhythms, and awake states by somewhat longer epochs of theta. The majority of MTLE seizures arise in NREM or awake states with very few starting during REM sleep. The thickness of each arrow pointing to the large-amplitude seizure (purple) indicates the relative probability of seizure onset from each state. $\boldsymbol{B}$, Schematic summary of the main findings of Sedigh-Sarvestani et al. (2014). Left, Injection of tetanus toxin into the rat hippocampus leads to chronic seizures and is thought to provide a good model of human MTLE. Right, As in humans, rodent hippocampal LFP during NREM sleep is characterized by occasional sharp-waveripple complexes. However, unlike human REM sleep, continuous and pronounced hippocampal theta oscillations are seen during rodent REM sleep. Awake periods in rats show either wide-band desynchronized activity (data not shown) or long-lasting bursts of continuous theta rhythms (shown in red). In this rat model, seizures are most likely to arise from REM sleep, less likely to arise from awake-theta periods and very unlikely to arise from NREM sleep. C, Left, Injections of tetanus toxin into different parts of the neocortex can be used to create models of focal neocortical epilepsy. Right, Schematic neocortical LFPs during different sleepwake states are shown. NREM sleep is characterized by large 1-4 Hz oscillations in the neocortex. Depending on the neocortical region injected, REM sleep and awake periods may or may not show prominent neocortical theta oscillations. Quantifying the likelihood of seizure onset in these various models can help to dissociate the relative contributions of REM sleep and theta rhythms in facilitating seizure onset.

it is difficult to disentangle the relative contributions of the REM state and the accompanying theta oscillations in creating an epileptogenic environment. Does the unique combination of neuromodulators released during REM sleep (e.g., increased acetylcholine and decreased serotonin/norepinephrine) facilitate seizure onset? Or does the unique excitation-inhibition balance reflected by hippocampal theta oscillations allow a seizure to result? Future experiments using tetanus toxin injections in the neocortex could help to resolve this debate. Tetanus toxin injected in neocortical structures is already a well established model of focal neocortical epilepsy (Benke and Swann,
2004). Also, distinct neocortical regions differentially express theta oscillations during distinct sleep-wake states: for example, during REM sleep in cats, theta oscillations are visible in the perirhinal cortex but not in posterior parietal cortex (Collins et al., 1999). Repeating SedighSarvestani et al.'s (2014) systematic quantification of pre-seizure states in animals receiving tetanus toxin injections in these two brain regions would help to test whether REM sleep can preferentially lead to seizures in the absence of local theta oscillatory dynamics (Fig. 1C). The result would shed important light on the mechanisms underlying seizure generation from the REM state in the tetanus toxin 
model of epilepsy. If brain regions lacking theta during REM sleep do not show the same increased propensity for REMinduced focal seizures, then this will suggest that the REM state is not inherently epileptogenic in the tetanus toxin model. Instead, the focus could shift to understanding the precise circuitry and neuromodulatory differences (e.g., subcortical cholinergic projections) between regions that show theta during REM sleep and those that do not (Brown et al., 2012). These differences would offer important clues to the mechanisms underlying seizure initiation.

Although the tetanus toxin model is a valuable tool to better understand, and eventually treat, human epilepsy, several studies have shown that when human temporal lobe seizures arise from sleep, they are far more likely to arise from NREM than REM sleep: NREM seizure rates were found to be two to six times higher than REM seizure rates $(\mathrm{Ng}$ and Pavlova, 2013). Why are the human observations so different from those found by Sedigh-Sarvestani et al. (2014)? As the authors point out, one crucial difference between humans and rodents is that human REM sleep is accompanied by only occasional, short bursts of hippocampal theta oscillations (Cantero et al., 2003) rather than the pronounced, continuous REM theta seen in the rodent hipoocampus (Fig. 1A). Thus, in the human hippocampus, as in some rodent neocortical areas, there is a partial disconnect between REM sleep and theta rhythms. This further emphasizes the importance of studying the effect of tetanus toxin injections into rodent brain regions that do not express theta during REM sleep (Fig. 1C). Moreover, there is an important similarity between human and rodent sleep: in both species, sleep deprivation leads to in- creased cortical theta rhythms (Brown et al., 2012) and also results in an increased likelihood of seizures (Foldvary-Schaefer and Grigg-Damberger, 2006). Although the causal relationship between sleepdeprivation-induced theta and seizure propensity has not yet been studied, this suggests that the disparate sleep-wake correlates of MTLE seizures in humans versus rats might be reconcilable. Since the human hippocampus displays shorter and rarer bursts of REM theta, seizures may be less likely to arise from this state in humans than in rats. NREM sleep may be less epileptogenic than periods of REM sleep accompanied by theta oscillations, but more epileptogenic than REM sleep when theta is absent; this might explain why a greater number of sleep seizures arise from NREM sleep in humans. These possibilities can be tested using human intracranial recordings from epilepsy patients by quantifying the amount of theta activity present before the onset of MTLE seizures.

The results presented by SedighSarvestani et al. (2014) raise several fundamental questions. In particular, since seizures can arise from a variety of brain states and rhythms, does this mean that the neuronal mechanisms underlying seizure initiation vary significantly based on the preceding brain state and rhythm? Or is there a unifying set of principles that can explain why similar seizures arise from differing sleep-wake states and LFP rhythms? By recording and optogenetically manipulating the activity in both the epileptic focus and the brainstem, future studies will be able to better understand the mechanisms that link sleep, rhythms, and seizures in different rodent models of epilepsy. Answering these questions will help get us closer to better un- derstanding, predicting, and hopefully treating human epilepsy.

\section{References}

Annegers JF (2001) The epidemiology of epilepsy. In: The treatment of epilepsy: principles and practice, 3rd Edition (Wyllie E, ed), pp 131-138. Philadelphia: Lippincott Williams and Wilkins.

Benke TA, Swann J (2004) The tetanus toxin model of chronic epilepsy. Adv Exp Med Biol 548:226-238. CrossRef Medline

Brown RE, Basheer R, McKenna JT, Strecker RE, McCarley RW (2012) Control of sleep and wakefulness. Physiol Rev 92:1087-1187. CrossRef Medline

Buzsáki G (2005) Theta rhythm of navigation: link between path integration and landmark navigation, episodic and semantic memory. Hippocampus 15:827-840. CrossRef Medline

Cantero JL, Atienza M, Stickgold R, Kahana MJ, Madsen JR, Kocsis B (2003) Sleep-dependent theta oscillations in the human hippocampus and neocortex. J Neurosci 23:10897-10903. Medline

Chang BS, Lowenstein DH (2003) Epilepsy. N Engl J Med 349:1257-1266. CrossRef Medline

Collins DR, Lang EJ, Paré D (1999) Spontaneous activity of the perirhinal cortex in behaving cats. Neuroscience 89:1025-1039. CrossRef Medline

Foldvary-Schaefer N, Grigg-Damberger M (2006) Sleep and epilepsy: what we know, don't know, and need to know. J Clin Neurophysiol 23:4-20. CrossRef Medline

Litt B, Lehnertz K (2002) Seizure prediction and the preseizure period. Curr Opin Neurol 15: 173-177. CrossRef Medline

Ng M, Pavlova M (2013) Why are seizures rare in rapid eye movement sleep? Review of the frequency of seizures in different sleep stages. Epilepsy Res Treat 2013:932790. CrossRef Medline

Sedigh-Sarvestani M, Thuku GI, Sunderam S, Parkar A, Weinstein SL, Schiff SJ, Gluckman BJ (2014) Rapid eye movement sleep and hippocampal theta oscillations precede seizure onset in the tetanus toxin model of temporal lobe epilepsy. J Neurosci 34:1105-1114. CrossRef Medline

Temkin O (1994) The falling sickness: a history of epilepsy from the Greeks to the beginnings of modern neurology. Baltimore: Johns Hopkins UP. 